\title{
Stage IVA2
}

National Cancer Institute

\section{Source}

National Cancer Institute. Stage IVA2. NCI Thesaurus. Code C141345.

A mycosis fungoides/Sezary syndrome stage defined as follows: T1-4, N3, M0, B0-2. T1: Limited patches, papules, and/or plaques covering less than $10 \%$ of the skin surface. 12 : Patches, papules, or plaques covering $10 \%$ or more of the skin surface. T3: One or more tumors (equal or greater than $1 \mathrm{~cm}$ in diameter). T4: Confluence of erythema covering $80 \%$ or more of body surface area. N3: Clinically abnormal peripheral lymph nodes; histopathology Dutch grades 3-4 or NCI LN4; clone positive or negative. M0: No visceral organ involvement. B0: Absence of significant blood involvement: $5 \%$ or less of peripheral blood lymphocytes are atypical (Sezary cells). B1: Low blood tumor burden: more than $5 \%$ of peripheral blood lymphocytes are atypical (Sezary cells) but does not meet the criteria of B2. B2: High blood tumor burden: 1,000 per microliter or more Sezary cells with positive clone. (AJCC 8th ed.) 